\title{
AFECÇÕES OCULARES EM CRIANÇAS DE 2 A 8 ANOS DA REDE PÚBLICA MUNICIPAL DE PIRACICABA - SP
}

\author{
OCULAR AFFECTIONS IN 2 TO 8 YEAR-OLD CHILDREN AT PIRACICABA CITY -SP
}

Paulo Cesar Gaiotto ${ }^{1}$; Walberto Passos Júnior ${ }^{2}$, Silvana Artioli Schellini ${ }^{3}$; Cláudia Akemi Shiratori ${ }^{4} \&$ Carlos Roberto Padovani ${ }^{5}$

${ }^{1}$ Professor Voluntário. ${ }^{2}$ Residente. ${ }^{3}$ Docente. Departamento de Oftalmologia, Otorrinolaringologia e Cirurgia de Cabeça e Pescoço ${ }^{4}$ Pósgraduanda. Faculdade de Medicina de Botucatu-UNESP. ${ }^{5}$ Docente. Departamento de Bioestatística. Instituto de Biociências - UNESP CoRRESPondÊnCIA: Silvana Artioli Schellini. Departamento de OFT/ORL/CCP - Faculdade de Medicina de Botucatu - UNESP $18618-970$ Botucatu - SP - Brasil - e-mail: sartioli@fmb.unesp.br

GAIOTTO PC; PASSOS JÚNIOR W; SCHELLINI AS; SHIRATORICA \& PADOVANICR. Afecções oculares em crianças de 2 a 8 anos da Rede Pública Municipal de Piracicaba - SP. Medicina, Ribeirão Preto, 35: 487-491, out./dez. 2002.

RESUMO: Modelo do Estudo: observacional. Objetivo: determinar a freqüência de ametropias e outros distúrbios oculares em uma população de crianças de 2 a 8 anos de idade, das escolas públicas municipais de Piracicaba - SP. Pacientes e Método: durante o ano letivo de 2000, foram encaminhadas para exame oftalmológico, completo 1001 crianças com idade entre 2 e 8 anos, matriculadas nas escolas públicas municipais de Piracicaba-SP. A medida de acuidade visual (tabela E de Snellen) foi previamente avaliada por professoras treinadas. As crianças que apresentaram acuidade visual menor ou igual a 0,8, bem como queixas ou sinais oftalmológicos, foram selecionadas para exame. Resultados: dentre 1001 crianças selecionadas, 5,09\% não compareceram ao exame, foram submetidas a exame oftalmológico completo 950 crianças. Detectaram-se ametropias em $70,84 \%$, sendo o astigmatismo hipermetrópico composto e a hipermetropia, simples os erros refracionais prevalentes (49,62\% e $32,98 \%$, respectivamente). Anisometropia foi observada em $1,78 \%$ dos examinados. Outras afecções oculares foram constatadas em 10,21\% das crianças; estrabismo (3,36\%), alterações palpebrais, conjuntivite alérgica, dacrioestenose congênita, atrofia óptica, coriorretinite e glaucoma congênito. Conclusão: a freqüência dos problemas oculares, observados indica quão importante é a realização de campanhas do tipo da apresentada para a redução dos índices de cegueira prevenível, no Brasil.

Unitermos: Cegueira; prevenção. Criança. Erros de Refração. Saúde Ocular.

\section{INTRODUÇÃO}

Estima-se, em termos globais, a existência de cerca de 38 milhões de cegos, bem como de 110 milhões de deficientes visuais com risco acentuado de cegueira $^{(1)}$.

Levantamentos da OMS mostram que cerca de 500.000 crianças tornam-se cegas a cada ano $^{(2)}$. Embora tenha ocorrido enorme evolução de âmbito terapêutico em Oftalmologia, a cegueira prevenível continuará sendo comum, principalmente entre os países menos favorecidos, a menos que sejam realizados esforços internacionais no sentido de erradicá-la ${ }^{(1)}$.
Aproximadamente $20 \%$ das crianças em idade escolar apresentam algum tipo de distúrbio ocular ${ }^{(3)}$. As causas dos distúrbios são as mais variadas e podem estar vinculadas a fatores biológicos, sociais e ambientais $^{(4)}$.

Os erros de refração, não corrigidos são das principais causas de deficiência visual nas crianças do Brasil ${ }^{(5)}$, sendo, também, apontados entre os distúrbios oculares mais freqüentes, na América Latina ${ }^{(6)}$.

As moléstias oculares são responsáveis por grande impacto econômico e psicossocial ${ }^{(7)}$. São capazes de influir no rendimento escolar e na sociabilização da criança, requerendo ações precoces de identificação e tratamento ${ }^{(3)}$. 
As campanhas de prevenção da cegueira estão se multiplicando no Brasil. Porém, os programas oficiais de medida de acuidade visual funcionam para crianças que iniciam o primeiro grau e que têm 6 ou 7 anos. Até a idade escolar, pode passar desapercebida da família a maior parte das dificuldades visuais da criança ${ }^{(8)}$.

Embora existam controvérsias a respeito da necessidade de triagem oftalmológica para pré-escolares $^{(9)}$ há, segundo a OMS, grande relevância nos esforços educativos, realizados como parte de programas e projetos de promoção da saúde ocular, que visem ao aumento do controle sobre os determinantes da saúde visual ${ }^{(10)}$. As triagens visuais funcionariam nesse sentido.

E necessária a orientação dos professores no sentido de melhorar a compreensão a respeito do papel fundamental que as triagens desempenham nas ações de saúde ocular na escola , sugerindo a implantação e a efetivação de programas de saúde ocular em todo o sistema de ensino, visando desenvolver ações de prevenção da incapacidade visual e da cegueira, promoção e recuperação da saúde ocular ${ }^{(4)}$.

O presente estudo tem, como propósito, estabelecer o perfil epidemiológico de uma população de pré-escolares, previamente triados por professores, no que concerne a moléstias oculares.

\section{MATERIAL E MÉTODO}

Foram triadas para exame oftalmológico 1001 crianças matriculadas na Rede Pública Municipal de Piracicaba-SP, com idades entre 2 e 8 anos, no período do ano letivo de 2000. As crianças foram selecionadas pelas professoras, utilizando-se tabela de optótipos de Snellen. As professoras foram treinadas por um dos autores (PCG), sendo capacitadas para a realização do teste de acuidade visual pela Tabela de Snellen (letra E), assim como para o reconhecimento dos problemas oculares detectáveis por meio do exame externo, quais sejam, conjuntivite, estrabismo, causas de lacrimejamento.

O critério de seleção das crianças a serem examinadas consistiu em detecção de acuidade visual (AV) menor ou igual a 0,8 em um dos olhos, sem o uso de correção óptica ou diferença maior ou igual a duas linhas entre os dois olhos, além de queixas (astenopia, embaçamento visual, epífora, dentre outras) ou sinais oculares ("olho torto", hiperemia, secreção), que pudessem indicar a presença de alterações oculares .

Uma vez selecionadas, as crianças foram sub- metidas a exame oftalmológico completo, realizado por oftalmologistas da Rede Municipal de Atendimento de Piracicaba. O exame consistiu de exame externo, avaliação da motilidade ocular extrínseca, exame biomicroscópico, exame refracional objetivo sob ciclopegia (ciclopentolato- 1gota a cada $5 \mathrm{~min}$, por 3 vezes e intervalo de $60 \mathrm{~min}$ para início do exame ) e oftalmoscopia direta .

Para caracterização do diagnóstico de ametropias, o critério adotado foi o seguinte: hipermetropia , quando grau esférico fosse maior que $1 \mathrm{D}$; astigmatismo, quando o grau cilíndrico fosse maior que $0,50 \mathrm{D}$, sendo classificado em hipermetrópico, miópico (simples e compostos ) e misto; miopia, quando o grau esférico fosse superior a 0,50 D e emetropia, quando não houvesse satisfação de nenhum dos critérios acima. Foi definida como anisometropia a diferença de 2 dioptrias esféricas ou cilíndricas entre os dois olhos. As crianças que não foram consideradas emétropes e que apresentavam queixas visuais receberam a prescrição de correção óptica.

Os dados obtidos foram submetidos a análise estatística, descritiva.

\section{RESULTADOS}

Das 1001 crianças triadas para exame ocular, $5,09 \%$ não foram avaliadas por motivo de falta no dia marcado para o exame .

Das 950 crianças examinadas, $57,36 \%$ eram do sexo masculino.

No que concerne ao exame de acuidade visual, $35,36 \%$ crianças mostraram-se inaptas, não tendo sido considerado o resultado da aferição em função da imprecisão dos dados obtidos.

Dos 950 pré-escolares examinados, 70,84\% eram portadores de erros refracionais, enquanto $10,21 \%$ tinham outro tipo de afecção ocular quais sejam: estrabismo manifesto $(3,36 \%)$, conjuntivites alérgicas $(2,84 \%)$, alterações palpebrais $(1,36 \%)$, telecanto $(1,15 \%)$, dentre outras. (Tabela I)

Dos 29,15\% pacientes considerados emétropes, $8,22 \%$ apresentavam alguma outra moléstia ocular, enquanto $20,94 \%$ foram considerados absolutamente normais.

Dentre as 673 amétropes, 32,98\% apresentavam hipermetropia simples; $63,15 \%$ eram astigmatas, das quais a maioria $(49,62 \%)$, portadora de astigmatismo hipermetrópico, composto; $2,08 \%$ eram míopes e $1,78 \%$ revelaram-se anisométropes. (Tabela II) 
Tabela I: Distribuição da população examinada quanto aos distúrbios oculares

\begin{tabular}{|l|c|c|}
\hline Distúrbios Oculares & $\begin{array}{c}\mathbf{N}^{\mathbf{0}} \mathbf{d e} \\
\text { crianças }\end{array}$ & \% ( \\
\hline Emétropes & 277 & 29,15 \\
\hline Amétropes & 673 & 70,84 \\
\hline Estrabismo Manifesto & 32 & 3,36 \\
\hline Conjuntivite Alérgica & 7 & 2,84 \\
\hline Alterações Palpebrais & 13 & 1,36 \\
\hline Epicanto & 5 & 0,52 \\
\hline Ptose & 5 & 0,52 \\
\hline Blefarite & 2 & 0,21 \\
\hline Papiloma palpebral & 1 & 0,1 \\
\hline Telecanto & 11 & 1,15 \\
\hline Dacrioestenose congênita & 6 & 0,63 \\
\hline Nevo conjuntival & 1 & 0,1 \\
\hline Coloboma de íris, retina e & 1 & 0,1 \\
\hline nervo óptico & & 0,1 \\
\hline Nistagmo horizontal & 1 & 0,1 \\
\hline Ceratocone & 1 & 0,1 \\
\hline Coriorretinite & 2 & 0,21 \\
\hline Atrofia óptica & 1 & 0,1 \\
\hline Glaucoma congênito & 1 & 0,1 \\
\hline & & \\
\hline & & \\
\hline
\end{tabular}

\section{DISCUSSÃ̃O}

A perda da capacidade visual implica em detrimento da qualidade de vida, decorrente de restrições ocupacionais, econômicas, sociais e psicológicas ${ }^{(11)}$.

A realização de programas de avaliação oftalmológica, tanto em crianças como em adultos, pode reduzir significativamente, na comunidade, a incidência de cegueira prevenível ${ }^{(12)}$.

Um modelo efetivo de prevenção à cegueira consiste em disponibilidade de atendimento clínico, de profissionais oftalmologistas bem treinados e de estudos epidemiológicos de base populacional ${ }^{(13)}$. Estudos sobre a freqüência das doenças oculares têm sido largamente realizados no Brasil ${ }^{(14)}$.
A população estudada esteve compreendida na faixa etária entre 2 e 8 anos, com média de idade de 4,32 anos. Devido ao fato de o desenvolvimento visual se completar aos 7 anos, é muito importante que se avalie a acuidade visual de crianças na faixa etária de pré-escolares, quando a possibilidade de recuperação visual é maior.

"Screening" realizado em país europeu, em crianças com 1 ano de idade e, depois, aos 2 e 3 anos de idade, mostrou que a ambliopia estava presente em $40 \%$ das crianças com refração anormal ${ }^{(15)}$. Segundo outros autores, nos países desenvolvidos, como Estados Unidos e Suíça, são realizados sistematicamente exames periódicos nas crianças pré-escolares e escolares desde a década de $50^{(16)}$.

O desenvolvimento de programas de "screening" visual em escolas elementares deve ser enfatizado, tendo em mente a necessidade de detecção e tratamento precoce de erros refrativos e de outras moléstias oculares $^{(17)}$.

A participação do professor é fundamental, uma vez que a convivência diária com seus alunos permite-lhe a detecção de mudanças de comportamento ou aparência, que podem estar vinculadas a distúrbios visuais ${ }^{(18)}$. No presente estudo, as professoras foram os agentes responsáveis pela triagem, o que tem ocorrido na grande maioria dos estudos similares, tendo sido muito bem feita, uma vez que $79,05 \%$ das crianças selecionadas apresentaram algum tipo de distúrbio visual .

Tabela II: Distribuição da população submetida a exame oftalmológico quanto à prevalência de erros refrativos

\begin{tabular}{|l|c|c|}
\hline Ametropia & $\begin{array}{c}\mathbf{N}^{\mathbf{0}} \mathbf{d e} \\
\text { crianças }\end{array}$ & $\mathbf{\%}(\mathbf{n = ~ 6 7 3})$ \\
\hline Hipermetropia simples & 222 & 32,98 \\
\hline Astigmatismo & 425 & 63,15 \\
\hline Hipermetrópico simples & 30 & 4,45 \\
\hline Hipermetrópico composto & 334 & 49,62 \\
\hline Miópico simples & 11 & 1,63 \\
\hline Miópico composto & 21 & 3,12 \\
\hline Misto & 29 & 4,30 \\
\hline Miopia simples & 14 & 2,08 \\
\hline Anisometropia & 12 & 1,78 \\
\hline
\end{tabular}


A triagem das crianças deve ser muito criteriosa. O treinamento e a motivação da equipe possibilitam que a detecção dos problemas se faça de forma mais satisfatória. Além da triagem bem feita, há necessidade do envolvimento das crianças e dos pais para que haja aderência ao tratamento. No presente estudo, a taxa de não comparecimento para exame foi relativamente baixa: $5,09 \%$ das crianças triadas. Em outros, a taxa de não comparecimento foi mais alta, observando-se até $20,6 \%$ de ausência de comparecimento $^{(16)}$.

Além da Tabela de Snellen, testes muito simples permitem que a triagem seja mais fidedigna, separando também afecções importantes, como o estrabismo e outras doenças oculares.

Deve-se considerar que, na faixa etária de préescolares, o desenvolvimento neurológico pode não ser o suficiente para o entendimento da tabela de acuidade visual, o que foi constatado em $35,36 \%$ das crianças que compuseram esta amostra. Nestas crianças, o uso do olhar preferencial pode ser útil, sendo este um exame muito simples.

Não existe homogeneidade entre os critérios para a classificação das ametropias, assim como para a seleção dos pacientes para exame oftalmológico. Os critérios aqui utilizados para classificação das ametropias seguiram os padrões propostos pelo Conselho Brasileiro de Oftalmologia ${ }^{(3)}$ : hipermetropia, quando grau esférico $>+1,00 \mathrm{D}$, astigmatismo, quando grau cilíndrico $>0,50 \mathrm{D}$, e miopia, quando grau esférico > $0,50 \mathrm{D}$. Outros consideraram, como hipermetropia, grau esférico maior ou igual a $+3,00 \mathrm{D}$, como astigmatis- mo, grau cilíndrico maior ou igual a $0,75 \mathrm{D}$, e, como miopia, graus maiores ou iguais $\mathrm{a}-0,25 \mathrm{D}^{(17)}$.

Em nosso estudo, foram selecionadas para exame oftalmológico, completo aquelas crianças que apresentavam AV menor ou igual a 0,8 bem como queixas ou sinais oftalmológicos. Outros selecionaram as crianças com AV menor ou igual a 0,7 (17)

Na população estudada, $70,89 \%$ das crianças foram consideradas amétropes. Tal porcentagem é variável, segundo os diferentes estudos. Schimiti et al. ${ }^{(17)}$ observaram ametropias em $31,26 \%$ das crianças triadas. Já Schellini et al. ${ }^{(16)}$ detectaram erros de refração em $63,9 \%$ das crianças selecionadas. Uma causa para essas diferenças está nos diferentes critérios utilizados para classificação e seleção ${ }^{(19)}$.

Os erros hipermetrópicos (hipermetropia, astigmatismos hipermetrópicos) foram as ametropias prevalentes. Para outros, também os erros hipermetrópicos constituíram a maioria, em crianças pré-escolares ${ }^{(9,16,20)}$.

Reveste-se de grande importância o tratamento precoce de ametropias, a fim de ser reduzida a incidência de ambliopia em crianças ${ }^{(21)}$. Neste estudo, todas as crianças consideradas amétropes e que apresentavam queixas oculares, foram tratadas com a devida prescrição de correção óptica.

A prevalência de outras patologias oculares entre as crianças triadas está de acordo com o observado por outros autores.

A realização e a repetição periódica de campanhas do tipo da apresentada são muito importantes e, sem dúvida, estarão alterando os índices de prevalência de cegueira infantil, no Brasil.

GAIOTTOPC; PASSOS JÚNIOR W; SCHELLINIAS; SHIRATORICA \& PADOVANICR. Ocular affections in 2 to 8 year- old children at Piracicaba City - SP. Medicina, Ribeirão Preto, 35: 487-491, oct./dec. 2002.

ABSTRACT: Study model: observacional, retrospective. Objective: to determine the frequence of the ametropic errors and other ocular problems in children with 2 to 8 year-old at Piracicaba SP. Patients and Method: During the school year of 2000, 1001 children enrolled at the public schools of Piracicaba - SP, age ranged from 2 to 8 years old, were referred to complete ophthalmological exam. Visual acuity was previously determined using Snellen chart, applied by school teachers. Those children presenting visual acuity equal or less than 0,8 , visual complaints or visual disorders were selected to appointment. Results: 51 children (5,09\%) did not attended to examination. 950 children were submitted to complete ophthalmological exam. Ametropic errors were found $70,84 \%$ of the children. The most prevalent refractive errors were Hypermetropic Astigmatism (49,62\%) and Hypermetropia (32,98\%). Anisometropia was found in 1,78\% children. Other ocular disabilities accounted for $10,21 \%$ of the examined children, such as strabismus $(3,36 \%)$, eyelid changes, allergic conjunctivitis, congenital dacryostenosis, optic atrophy, corioretinitis and congenital glaucoma. Conclusion: The frequence of ocular problems observed let us to conclude the screening programs are valid surveys on decreasing rates of preventable blindness in our country.

UNITERMS: Blindness; prevention. Child. Refractive Errors. Eye Health. 


\section{REFERÊNCIAS BIBLIOGRÁFICAS}

1 - THYLEFORS B. Prevention of blindness-WHO's mission for vision. World Health Forum 19:53-59,1998.

2 - FOSTER A \& GILBERT C. Epidemiology of childhood blindness. Eye 6: 173-176, 1992.

3 - ALVES M R \& KARA-JOSÉ N. Campanha “Veja Bem Brasil". Manual de orientação. Conselho Brasileiro de Oftalmologia, 1998.

4 - ARMOND JE; TEMPORINI E R \& ALVES MR. Promoção da saúde ocular na escola: Percepções de professores sobre erros de refração. Arq Bras Oftalmol 64:395-400, 2001.

5 - KARA-JOSÉ N \& ALVES MR. Problemas oftalmológicos mais freqüentes em escolares. In: CONCEIÇÃO JAN. Saúde escolar: A criança, a vida, e a escola. Sarvier, São Paulo, p.195-203, 1994.

6 - VERDAGUER T J. One world, one voice, one vision. Arq Bras Oftalmol 61: 5-6, 1998.

7 - NÉGREL AD \& THYLEFORS B. The global impact of eye injuries. Ophthalmic Epidemiol 5:143-169, 1998.

8 - TEMPORINI ER. Prevenção de problemas visuais de escolares: Conduta de professores do sistema de ensino do Estado de São Paulo, Brasil. Rev Bras Saúde Esc 1:68$75,1990$.

9 - KASSMANN-KELLNER B; HEINE M; PFAN B; SINGE R \& RUPRECHT KW. Screening for amblyopia, strabismus and refractive abnormalities in 1030 Kindergartan children. Klin Monatsbl Augenheilkd 213: 166-173, 1998.

10 - WORLD HEALTH ORGANIZATION. Otawa charter for health promotion. WHO, Ontario, 1986.

11 - TEMPORINI E R \& KARA-JOSÉ N. Níveis de prevenção de problemas oftalmológicos. Arq Bras Oftalmol 58:189-192, 1995.

12 - JACKSON C \& GLASSON W. Prevention of visual loss. Screening in general pratice. Aust Fam Physician 27:150$153,1998$.
13 - JINGJI X; WENSHU M.; SHAOGHEN L; LEGHEN W; ZHONGYAO W; SIPING Z; QIANG Y \& MINGGUANG HNG. A model for blindness prevention. Yanke Xue Bao 13: 162163, 1997.

14 - MACCHIAVERNI FN; KARA-JOSÉ N; RUEDA G; PEREIRA VL; COSTA MN \& RANGEL FF. Levantamento oftalmológico em escolares da primeira a quarta série do primeiro grau na cidade de Paulínia-SP. Arq Bras Oftalmol 42: 289-294, 1979.

15 - INGRAM RM. The problem of screening children for visual defects. Br J Ophthalmol 61: 4-7, 1977.

16 - SCHELLINI S A; SHIMODA G A; HISANO C; UTYAMA LAC \& SILVA MRBM. Estudo da prevalência das afecções oculares nos pré-escolares da cidade de Botucatu-SP. Rev Bras Oftalmol 46:61-65, 1987.

17 - SCHIMITI RB; COSTA V P; GREGUI M JF; KARA-JOSÉ N \& TEMPORINI ER. Prevalence of refractive errors and ocular disorders in preschool and schoolchildren of Ibiporã - PR, Brazil (1989 to 1996). Arq Bras Oftalmol 64:379-384, 2001.

18 - GALOTTI O. Oftalmologia sanitária- conceituação. Rev Bras Oftalmol 34: 219-223, 1971.

19 - CORDONNIER M \& DRAMAIX M. Screening of abnormal levels of hyperopia in children: A non cycloplegic method with a hard held refractor. Br J Ophthalmol 82: 1260-1264 , 1998.

20 - RODRIGUEZ MA \& CASTRO-GONZALES M. Visual Health of school children in Medellin Antioquia, Colômbia. Bol Oficina Sanit Panam 119:11-14, 1995.

21 - LESUEUR L; CHAPOTOT E; ARNEJ L; PERRON-BUSCAIL A \& DENEUVILLE S. Predictability of amblyopia in ametropic children- a propos of 96 cases. J Fr Opththalmol 21: 415424, 1998.

Recebido para publicacao em 22/08/2002

Aprovado para publicacao em 27/12/2002 